\title{
Correspondence
}

\section{Interleukin production in juvenile chronic arthritis}

SIR, We read with interest the paper by Gilman and coworkers who showed that in rats with adjuvant-induced arthritis peritoneal macrophages produce higher amounts of interleukin 1 (IL-1), while splenic T cells produce less interleukin 2 (IL-2) than controls. ' We found comparable results when studying interleukin production by peripheral blood mononuclear cells in children with juvenile chronic arthritis (JCA) (unpublished data). We investigated a total of 28 children with JCA ranging in age from 3 to 16 years. Ten presented a systemic form, six a polyarticular, and twelve a pauciarticular form. Patients were divided according to clinical activity of the disease into three groups: active disease, partial remission, and remission. Twentyone age matched healthy children served as controls. Peripheral blood monocytes were isolated by adherence and stimulated in vitro with lipopolysaccharide: IL-1 in the supernatants was assessed by a thymocyte coproliferation assay. Peripheral blood lymphocytes were stimulated in vitro with phytohaemagglutinin, and IL-2 in the supernatants was quantified by an IL-2-dependent cell line.

Monocytes from patients with JCA produced significantly more IL-1 than controls $(17 \cdot 1 \pm 11 \cdot 1$ (SD) $v$ $10 \cdot 6 \pm 8.9$ (SD), $p<0 \cdot 05$ ), while lymphocytes from the same patients produced significantly less IL-2 than controls $(40.6 \pm 31.2$ (SD) $v 69.8 \pm 40.9$ (SD), $\mathrm{p}<0.01)$. These findings could not be explained by concurrent treatment. The greater IL-1 production was more evident in patients with active disease, whereas production of IL-2 was lowest in patients with complete remission. No major differences were observed among the three JCA subtypes. The similarities between our findings in JCA patients and the findings of Gilman et al. ${ }^{1}$ in adjuvant-induced arthritis suggest that modulation of interleukin production may be of relevance in the pathogenesis of JCA, and, together with the previous finding of reduced production of IL-2 in adult rheumatoid arthritis and systemic lupus erythematosus, ${ }^{23}$ indicates that aberrant regulation of interleukin cascade may be a common feature (probably secondary to different aetiologic factors) of some immunemediated diseases.

Department of Pediatrics.

G R BURGIO

University of Pavia,

A MARTINI

Policlinico S Matteo

27100 Pavia.

Italy

\section{References}

1 Gilman S C. Daniels J F. Wilson R E. Carlson R P. Lewis A J. Lymphoid abnormalities in rats with adjuvant-induced arthritis.

I. Mitogen responsiveness and lymphokine synthesis. Ann Rheum Dis 1984: 43: 847-55.

2 Miyasaka N. Nakamura T. Russell I J. Talal N. Interleukin 2 deficiencies in rheumatoid arthritis and systemic lupus erythematosus. Clin Immunol Immunopathol 1984: 31: 109-17.

3 Linker-Israeli M. Bakke A C. Kitridou R C. Gendler S. Gillis S. Horwitz D A. Defective production of interleukin 1 and interleukin 2 in patients with systemic lupus erythematosus (SLE). J Immunol 1983; 130: 2651-5.

\section{Hypogammaglobulinaemia and thrombocytopenia associated with sulphasalazine therapy in rheumatoid arthritis}

SIR, Hypogammaglobulinaemia has been reported as a complication of chrysotherapy in rheumatoid arthritis (RA) 12 but has not previously been reported after sulphasalazine (SASP) therapy. SASP is now becoming established as an effective second-line drug in RA with apparently few serious side effects. ${ }^{3-5}$ We report a patient in whom both hypogammaglobulinaemia and thrombocytopenia together with oedema and rash occurred with this drug.

\section{Case history}

A 61-year-old lady with seropositive, erosive nodular RA had an excellent clinical response to SASP $2 \mathrm{~g}$ daily, with a concomitant fall in her erythrocyte sedimentation rate (ESR). Treatment was continued for 18 weeks at which time she developed clinical and laboratory abnormalities. She presented with oedema and a rash on her face and lower legs. The rash was erythematous, pruritic, and non-purpuric. The platelet count had fallen to $76000 / \mathrm{mm}^{3}$ $\left(76 \times 10^{9} / 1\right)$ (Fig. 1), but white blood cell count was normal. IgG had fallen from the initial level of 8.19 to $4.95 \mathrm{~g} / \mathrm{l}$, IgA from $2 \cdot 16$ to $0.39 \mathrm{~g} / \mathrm{l}$, IgM from 2.75 to $0.34 \mathrm{~g} / \mathrm{l}$, and the Rose-Waaler titre from $1 / 156$ to $1 / 8$, and serum albumin was unchanged. She had no proteinuria. Sulphasalazine was stopped, and one week later both rash and oedema had subsided. During the following three weeks the serum IgG level fell further to $1.9 \mathrm{~g} / \mathrm{l}$, though serum IgA rose to $0.63 \mathrm{~g} / \mathrm{l}$ and $\mathrm{IgM}$ to $0.47 \mathrm{~g} / \mathrm{l}$. Despite the immunodeficiency and her past history of chest infections she continued to be well and her arthritis remained in remission. After this the serum immunoglobulin levels and platelet count increased and at three months all were normal (Fig. 1). She continued the same non-steroidal anti-inflammatory agent (fenbufen) throughout the time reported.

Hypogammaglobulinaemia, thrombocytopenia, oedema, and a non-purpuric rash occurred after 18 weeks of SASP therapy. These resolved after stopping the drug, thus implicating SASP as a causative factor. Our patient had previously taken various non-steroidal antiinflammatory drugs without side effects but no second-line 
agents and continued fenbufen throughout the time reported. Thrombocytopenia is a recognised complication of other second-line drugs, including gold and penicillamine. It has been reported (as part of pancytopenia) in association with SASP therapy in inflammatory bowel disease $^{67}$ but not in RA. It is thought to be due to the sulphapyridine moiety. Hypogammaglobulinaemia has
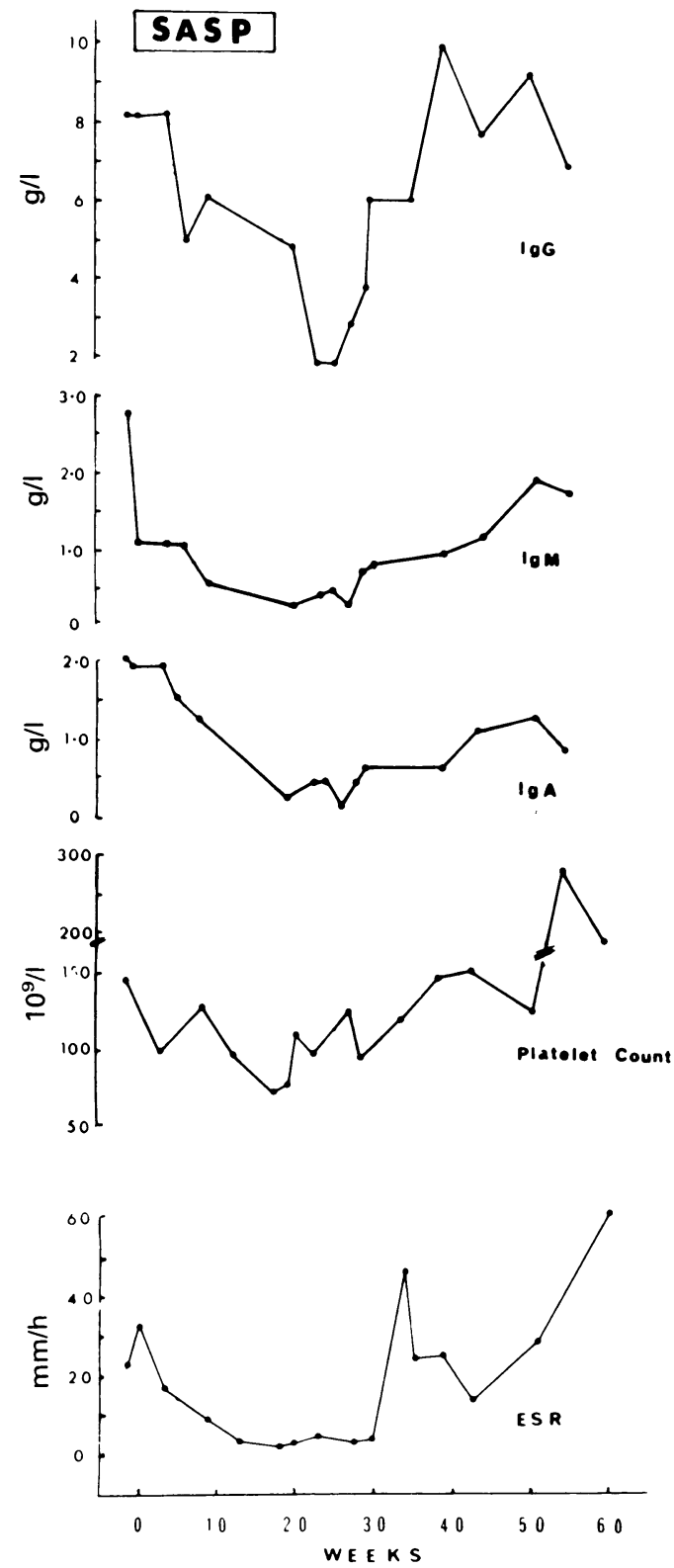

Fig. 1 Changes in serum levels of immunoglobulin $G, M$. and $A$, platelet count, and ESR during treatment with sulphasalazine. been reported in RA patients treated with chrysotherapy, ${ }^{12}$ and selective IgA deficiency has beer recorded in RA patients receiving not only gold or; penicillamine $^{\gamma}$ but also SASP." Although the serun immunoglobulin levels returned to normal in our patienf within three months, in some of those treated with golf therapy the immunodeficiency persisted and even require

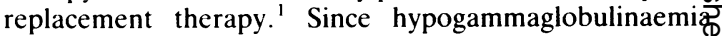
may be associated with infection and thrombocytopeniฉ with haemorrhage, SASP therapy in RA may not be quitछ̈ as safe as previously thought. Long term monitoring todetect and reverse these adverse reactions is clearly important with SASP treatment as it is with the other slow acting antirheumatic drugs.

Rheumatism Research Wing,

The Queen Elizabeth Hospital,

Birmingham B15 2TH

Morriston Hospital,

Heol Maes Eglwys,

Cwmrhydyceirn.

Swansea,

Wales

\section{References}

1 So A K L, Peskett S A, Webster A B. Hypogammaglobul naemia associated with gold therapy. Ann Rheum Dis 1984:43: 581-2.

2 Hunter T. Hypogammaglobulinaemia associated with therapy. Ann Rheum Dis 1985; 44: 212.

3 Pullar T. Capell H. Sulphasalazine: a 'new' antirheumatic d. Br J Rheum 1984: 23: 26-34.

4 Farr M. Tunn E. Crockson A P. Bacon P A. The long term effects of sulphasalazine in the treatment of rheumatoid arthrit and a comparative study with penicillamine. Clin Rheumat 1984: 3: 473-82.

5 Bax D E. Amos R S. Sulphasalazine: a safe, effective agent fo prolonged control of rheumatoid arthritis. A comparison wit sodium aurothiomalatc. Ann Rheum Dis 1985; 44: 194-9.

6 Collins J R. Adverse reactions to salicylazosulphapyridine (Azulfidine) in the treatment of ulcerative colitis. South Medळ 1968: 61: 354-8.

7 Van Hees P A M. Clinical and pharmacological aspects of sulphasalazine. The Netherlands: Kathalieke Universiteit to Nijmegen, 1980: 178-80. (MD thesis).

8 Johns P. Felix-Davies D D. Hawkins C F, et al. IgA deficiencry in patients with rheumatoid arthritis treated with to penicillamine or gold. Ann Rheum Dis 1978; 37: 289.

9 Delamere J P. Farr M. Grindulis K A. Sulphasalazine induced selective IgA deficiency in rheumatoid arthritis. Br Med J 1983 286: $1547-8$.

\section{Synovial fluid mast cells}

SIR, The paucity of mast cells in inflammatory synoviâ fluids reported by Freemont and Denton ${ }^{1}$ is not unex pected. The mast cell is primarily a tissue cell, though it conceivable thi $t$ its circulatory counterpart, the basophif may be found in greater numbers in inflammatory synovia? fluids. Are the authors certain that these scanty, granulao cells were not basophils? Whereas lavage of roder $\vec{\Phi}$ peritoneal membranes is a rich source of mast cells. I have 